\title{
Residuos de ivermectina en tejido hepático de alpacas (Vicugna pacos) en un matadero de Arequipa - Perú, 2019
}

\author{
Ivermectin residues in alpaca liver tissue (Vicugna pacos) in an \\ abattoir in Arequipa - Peru, 2019
}

\author{
Efraín Cárdenas ${ }^{1}$, Carlos Shiva ${ }^{2}$, Elizabeth Hinostroza ${ }^{3}$, Daphne León ${ }^{1}$, \\ Néstor Falcón ${ }^{1}$
}

\section{Resumen}

El objetivo del estudio fue determinar la presencia de residuos de ivermectina en tejido hepático de alpacas sacrificadas en un matadero de la provincia de Caylloma, departamento de Arequipa - Perú. Se recolectaron 50 muestras de hígados que fueron evaluadas mediante la técnica de ELISA competitiva con un kit comercial. Además, se recopiló información sobre la procedencia de las alpacas (Challhuanca, Tocra); edad (menos de 2 años, entre 2-3 años, más de 3-4 años y mayor de 4 años); sexo (macho o hembra) y raza (Huacaya o Suri). Se encontró $10 \%$ de hígados positivo para residuos de ivermectina con valores que estuvieron por encima del Límite Máximo de Residuos establecido por el Codex Alimentarius para tejido hepático de alpacas $(15 \mu \mathrm{g} / \mathrm{kg})$. Las muestras positivas contenían niveles entre 17.28 a $79.10 \mu \mathrm{g} / \mathrm{kg}$ de residuos de ivermectina. Se encontró resultados positivos para muestras de hígado de diferentes procedencias, gru-

1 Laboratorio de Epidemiología y Salud Pública en Veterinaria, Facultad de Medicina Veterinaria y Zootecnia, Universidad Peruana Cayetano Heredia, Lima, Perú

${ }^{2}$ Laboratorio de Nutrición Animal e Inocuidad Alimentaria, Facultad de Medicina Veterinaria y Zootecnia, Universidad Peruana Cayetano Heredia, Lima, Perú

${ }^{3}$ Laboratorio de Fisiología y Farmacología Veterinaria, Facultad de Medicina Veterinaria y Zootecnia, Universidad Peruana Cayetano Heredia, Lima, Perú

${ }^{4}$ E-mail: nestorfalcon@upch.pe; https://orcid.org/0000-0003-4144-0494

Recibido: 15 de septiembre de 2020

Aceptado para publicación: 4 de marzo de 2021

Publicado: 23 de junio de 2021

CLos autores. Este artículo es publicado por la Rev Inv Vet Perú de la Facultad de Medicina Veterinaria, Universidad Nacional Mayor de San Marcos. Este es un artículo de acceso abierto, distribuido bajo los términos de la licencia Creative Commons Atribución 4.0 Internacional (CC BY 4.0) [https:// creativecommons.org/licenses/by/4.0/deed.es] que permite el uso, distribución y reproducción en cualquier medio, siempre que la obra original sea debidamente citada de su fuente original 
po etario, sexo y raza, sin encontrar asociación significativa con la presencia de residuos de ivermectinas. Los resultados encontrados sugieren la necesidad de implementar estrategias de control de residuos a nivel de los mataderos en busca de mejorar las condiciones de inocuidad de los alimentos destinados a consumo humano.

Palabras clave: ELISA, antiparasitario, avermectinas, hígado

\section{AbSTRACT}

The objective of the study was to determine the presence of ivermectin residues in liver tissue of alpacas in an abattoir in the province of Caylloma, department of Arequipa, Peru. In total, 50 liver samples were collected and evaluated by competitive ELISA technique with a commercial kit. In addition, information was collected on the origin of the alpacas (Challhuanca, Tocra); age (less than 2 years, between 2-3 years, more than 3-4 years, and more than 4 years); sex (male or female) and breed (Huacaya or Suri). Results showed that $10 \%$ of livers were found to be positive for ivermectin residues with values that were above the Maximum Residue Limit established by the Codex Alimentarius for alpaca liver tissue $(15 \mu \mathrm{g} / \mathrm{kg})$. Positive samples contained levels between 17.28 to $79.10 \mu \mathrm{g} / \mathrm{kg}$ of ivermectin residues. Positive results were found for liver samples from different origin, age group, sex and breed, without finding a significant association with the presence of ivermectin residues. The results found suggest the need to implement residue control strategies at the slaughterhouse level to improve the safety conditions of food for human consumption.

Key words: ELISA, antiparasitic, avermectins, liver

\section{INTRODUCCIÓN}

La producción de la fibra y carne representa la principal fuente de ingresos económicos para los criadores de camélidos sudamericanos domésticos (alpaca y llama), estimándose que representa alrededor del $75 \%$ de ingresos anuales de 150000 familias en situaciones de pobreza y pobreza extrema que se dedican a esta actividad (Fernández-Baca, 2005; De los Ríos, 2010).

La explotación de camélidos sudamericanos enfrenta una serie de problemas sanitarios como la presencia de enfermedades parasitarias, entre ellas la parasitosis gastrointestinal, seguido en orden de importancia por la sarna, atribuyéndose a esta última el
95\% de pérdidas económicas causadas por ectoprásitos (Casas et al., 2006). Para enfrentar esta problemática, los productores utilizan fármacos a base de avermectina como método preventivo o tratamiento debido a su efectividad contra nematodos y ectoparásitos (Fernández-Baca, 2005; Condemayta et al., 2014).

La ivermectina (IVM) provoca una afluencia de iones $\mathrm{Cl}$ en la membrana celular de los invertebrados mediante la activación de los canales iónicos regulados por GABA (ácido gamma aminobutírico). La hiperpolarización resultante conduce al bloqueo de la estimulación pos-sináptica con la consecuente parálisis muscular y muerte de los parásitos (Plumb, 2010). Este fármaco se 
utiliza en Perú desde 1983, y debido a que otros productos como los piretroides sintéticos y organofosforados son moderadamente más tóxicos y menos eficaces, reemplazó su uso en diversas especies como camélidos sudamericanos, ovejas y ganado bovino $(\mathrm{Ca}-$ sas et al., 2006).

La detección de ivermectina en los alimentos se puede realizar mediante la técnica de Cromatografía Liquida de Alta Eficiencia (HPLC) que se caracteriza por su rapidez, alta sensibilidad y automatización; sin embargo, se requiere de una inversión elevada y personal con amplia experiencia para su funcionamiento (Danaher et al., 2006), mientras que la prueba de ELISA es mucho más económica y sencilla, y permite cuantificar residuos que se correlacionan estrechamente con las determinadas por HPLC $(\mathrm{r}=0.99)$ (Shi et al., 2006; Lailany et al., 2016).

La administración de ivermectina a especies de consumo humano es potencialmente riesgosa, porque puede encontrarse en tejidos comestibles, leche y otros derivados. Por ello, la Agencia Europea de Medicamentos estableció los límites máximos de residuos de IVM en la Unión Europea, con valores de $100 \mu \mathrm{g} \mathrm{kg}^{-1}$ en grasa e hígado y $30 \mu \mathrm{g} \mathrm{kg}^{-1}$ en riñón para todos los mamíferos productores de alimentos, mientras que la normativa de Codex Alimentarius establece $15 \mu \mathrm{g} \mathrm{kg}^{-1}$ para hígado de ovinos y rumiantes menores con el fin de garantizar que los residuos encontrados en los alimentos estén por debajo de los niveles perjudiciales para los consumidores (EMA, 2014; Codex Alimentarius, 2018). Según estos valores, los periodos de espera después de la inyección subcutánea se establecieron recientemente en Europa entre 49 y 60 días (Escribano et al., 2012).

En el Centro Poblado Challhuanca y anexos aledaños, el $90 \%$ de habitantes se dedican a la crianza de camélidos sudamericanos y el uso de antiparasitarios es una práctica común con el riesgo que no se respete los periodos de retiro (Condori y Ccorimanya,
2018). Por ello, el objetivo del estudio fue determinar la presencia de residuos de IVM en tejido hepático de alpacas (Vicugna pacos) sacrificadas en un matadero de Caylloma, departamento de Arequipa en el año 2019.

\section{Materiales y Métodos}

La investigación transversal y descriptiva recolectó muestras de hígado en un matadero de camélidos sudamericanos ubicado en el Centro Poblado Challhuanca, provincia Caylloma, departamento de Arequipa, Perú. El diagnóstico se realizó en la Facultad de Medicina Veterinaria y Zootecnia de la Universidad Peruana Cayetano Heredia (FAVEZ-UPCH).

El tamaño de muestra fue determinado con el programa WinEpi (Working in Epidemiology) utilizando la fórmula de prevalencia límite con un tamaño de población $\mathrm{N}=25319$ (INEI, 2012). Se trabajó con una prevalencia mínima de detección del 5.97\% (Barbieri, 2014) y nivel de confianza del 95\%, obteniendo un resultado de $n=49$.

La selección de la muestra se realizó con base a un muestreo sistemático. Se seleccionó una de cada cinco alpacas de acuerdo con el orden de ingreso al matadero durante el periodo julio - agosto de 2019. Se recopiló la siguiente información: lugar de procedencia de las alpacas (Challhuanca, Tocra); edad ( $<2$ años $=$ diente de leche, entre 2-3 años $=$ dos dientes, 3-4 años $=$ cuatro dientes, $>4$ años = boca llena); sexo (macho o hembra) y raza (Huacaya o Suri). La muestra recolectada fue tejido hepático de aproximadamente $100 \mathrm{~g}$ del lóbulo derecho utilizando hojas de bisturí estéril. Las muestras fueron colocadas en bolsas estériles (Nasco Whirl-Pak $\left.{ }^{\circledR}\right)$, rotuladas y almacenadas entre -10 y $-17^{\circ} \mathrm{C}$.

Las muestras fueron transportadas a la ciudad de Lima por vía terrestre dentro de una nevera portátil con gel refrigerante; don- 
de fueron congeladas a le llagada al Laboratorio de Nutrición Animal e Inocuidad Alimentaria de la FAVEZ-UPCH para su procesamiento y análisis. La cuantificación de residuos se realizó mediante un kit comercial de ELISA (MaxSignal ${ }^{\circledR}$ Avermectin \#1073-01B), siguiendo las indicaciones del fabricante PerkinElmer Company. Esta herramienta ha sido validada previamente por otros estudios para evaluar residuos de IVM en muestras de hígado y contaba con los siguientes controles: negativo, $0.5,1.0,2.0,4.0$ y $8.0 \mathrm{ng} / \mathrm{ml}$.

Las muestras de hígado se homogenizaron individualmente en mortero de porcelana. Se transfirió $2 \mathrm{~g}$ de cada muestra a tubos Falcon de $12 \mathrm{ml}$, a los cuales se agregó 6 $\mathrm{ml}$ de acetonitrilo grado HPLC con $2.5 \mathrm{ml}$ de tampón de equilibrio de avermectina $1 \mathrm{x}$ y se agitaron en el vórtex por 3 min a velocidad máxima. Luego se añadió $200 \mu 1$ de tampón de extracción a cada tubo y se centrifugaron a $8960 \mathrm{rpm}$ durante $10 \mathrm{~min}$. Se transfirió $200 \mu \mathrm{l}$ del sobrenadante a tubos Eppendorf y se realizó la evaporación con gas nitrógeno en baño María a $50{ }^{\circ} \mathrm{C}$. Los tubos fueron reconstituidos con $1 \mathrm{ml}$ de $35 \%$ etanol/tampón de extracción y agitados en vórtex durante $60 \mathrm{~s}$. Finalmente, se tomó $50 \mu 1$ de cada muestra y estándar para la realización de ELISA (Bioo Scientific Corporation, 2014).

El método de ELISA se basa en un ensayo competitivo, donde el fármaco de interés (IVM) estaba recubierto en los pocillos de la placa. Durante el análisis, la IVM presente en la muestra y el fármaco recubierto compiten por el anticuerpo primario luego de ser añadidas a la placa. En seguida, el anticuerpo secundario marcado con una enzima peroxidasa se dirige al complejo anticuerpo primario-fármaco recubierto. La intensidad de color resultante después de la adición del sustrato se expresa en una relación inversa con la concentración de IVM en la muestra
(Bioo Scientific Corporation, 2014). La cuantificación de residuos de IVM fue en base a los valores la Absorbancia Relativa (\%) y concentración de los estándares, con los cuales se elaboró una curva de calibración en Microsoft Excel 2013 que permitió obtener una ecuación logarítmica.

Los resultados del estudio fueron resumidos mediante estadística descriptiva en el programa Microsoft Excel, utilizando cuadros estadísticos para presentar los valores absolutos y relativos. Asimismo se evaluó la asociación de las variables (raza, sexo, edad, lugar de procedencia) con la presencia de residuos de IVM, mediante la prueba Exacta de Fisher. El estudio fue aprobado por el Comité Institucional de Ética para el uso de animales de la UPCH mediante la constancia N. ${ }^{\circ}$ 072-08-09.

\section{Resultados}

El 64\% $(n=32)$ de los animales provenían del Centro Poblado de Challhuanca, mientras que el restante $36 \%$ (18) del anexo Tocra. El 8\% (4) fueron menores de 2 años, $12 \%$ (6) de 2-3 años, $22 \%$ (11) de más de 3 a 4 años y $58 \%$ (29) mayores de 4 años. En cuanto al sexo, $68 \%$ (34) fueron hembras y $32 \%$ (16) machos; asimismo, 76\% (38) fueron de raza Huacaya y $24 \%$ (12) Suri.

Se elaboró una curva de calibración representando la absorbancia relativa y concentración de los estándares (Figura 1), a partir del cual se obtuvo la ecuación logarítmica con un coeficiente de determinación $\mathrm{R}^{2}=0.995$, donde la incógnita «y» fue reemplazada con los valores de absorbancia relativa de cada muestra y luego multiplicado por 20 (factor de dilución) para obtener la concentración de IVM. Estos resultados fueron corroborados mediante el programa MaxSignal ${ }^{\circledR}$ ELISA Analysis Programme suministrado por el fabricante. 


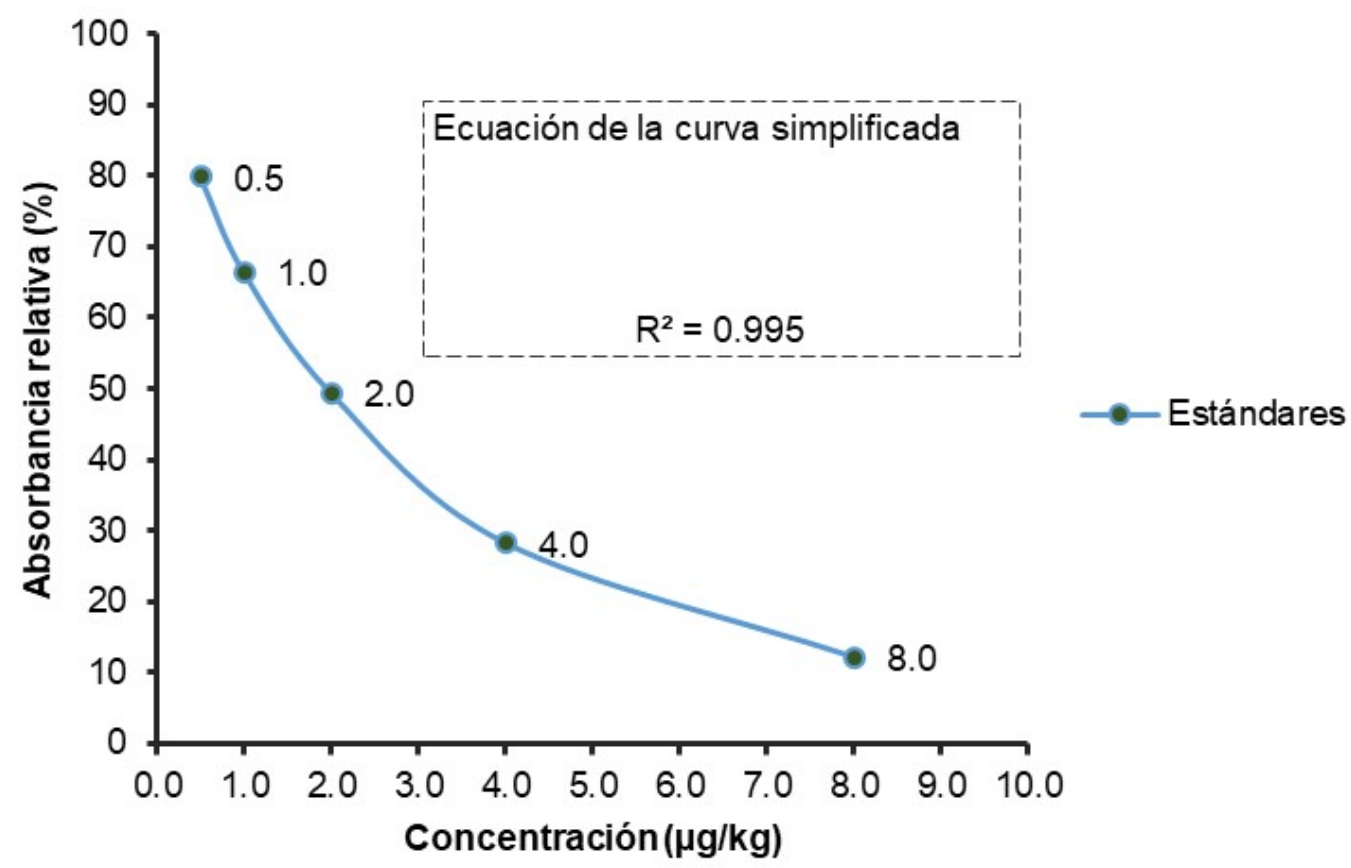

Figura 1. Curva de calibración de los estándares para cuantificar residuos de ivermectina en hígado de alpacas procedentes de la provincia de Caylloma, Arequipa, Perú en 2019. $\mathrm{R}^{2}$ : coeficiente de determinación; Y: valores de absorbancia relativa; $\mathrm{X}$ : concentración de IVM en $\mu \mathrm{g} / \mathrm{kg}$; e: constante matemática; 20: factor de dilución

El 10\% (5/50) de las muestras resultaron positivas para residuos del antiparasitario IVM con concentraciones que variaban entre 17.28 y $79.10 \mu \mathrm{g} / \mathrm{kg}$, superando los $15 \mu \mathrm{g} / \mathrm{kg}$ de Límite Máximo de Residuos (LMR) establecidos por el Codex Alimentarius para tejido hepático de alpacas (Figura 2).

Se reportó una mayor frecuencia relativa de muestras de hígados positivos a residuos de IVM en animales procedentes de Tocra, menores a 2 años, machos y de raza Huacaya, sin encontrarse diferencia significativa en las proporciones entre las categorías de las respectivas variables (Cuadro 1).

\section{Discusión}

El Centro Poblado de Challhuanca representa un modelo de la realidad de muchas poblaciones altoandinas del Perú, donde la principal actividad económica es la crianza de camélidos sudamericanos, en especial las alpacas. Factores como la limitada asistencia de servicio veterinario, uso de antiparasitarios de larga acción y la falta de registros de animales dosificados, entre otros, pueden representar un riesgo para la presencia de residuos de medicamentos como la IVM, que es usado en el tratamiento de la 


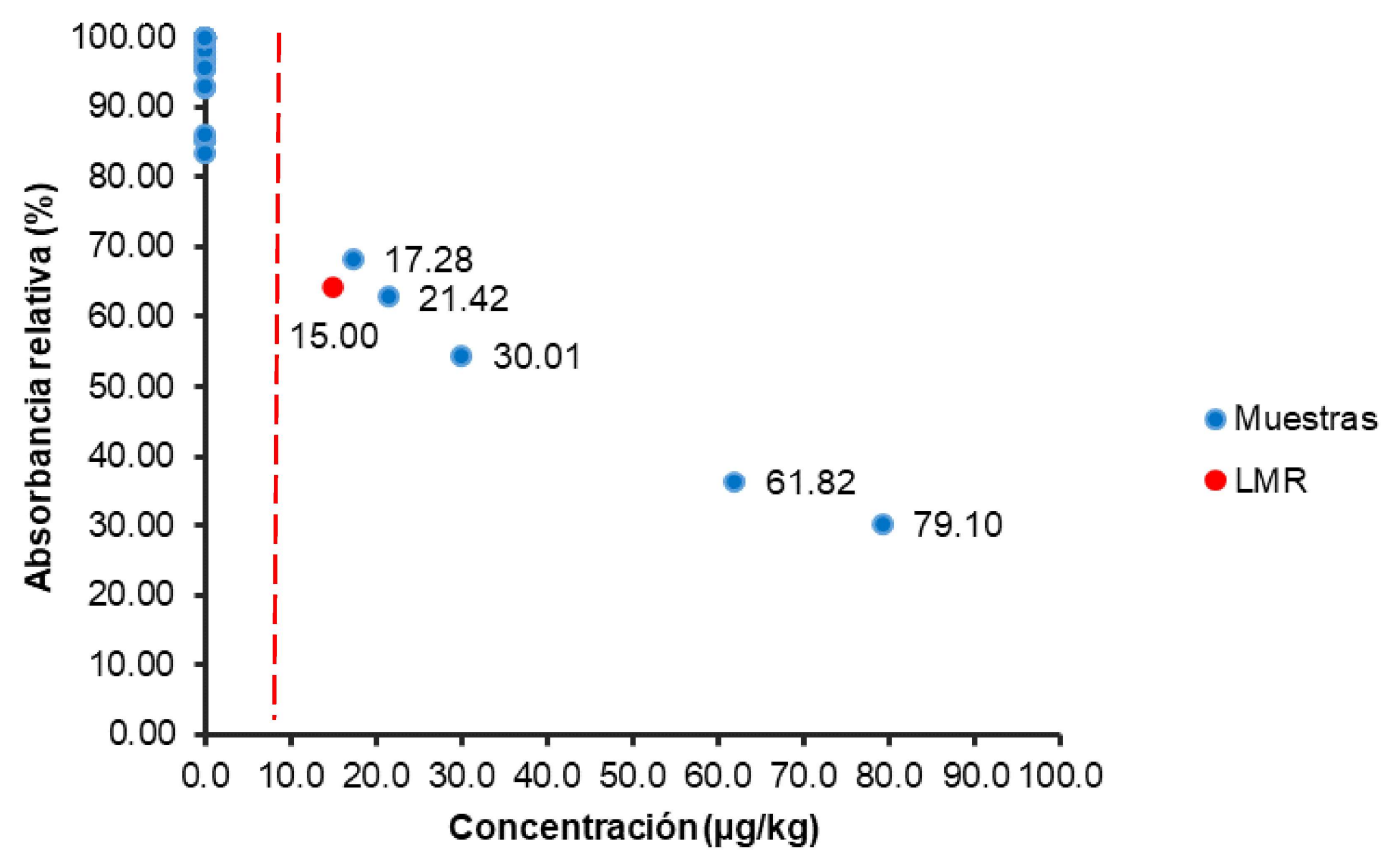

Figura 2. Cuantificación de residuos de ivermectina en muestras de hígado de alpacas de la provincia de Caylloma, Arequipa, Perú, año 2019, que sobrepasaron el Límite Máximo de Residuos (LMR) establecidos por el Codex Alimentarius (2018)

sarna, enfermedad parasitaria endémica en la zona. En ese sentido, CAPV (2016) menciona que el uso inadecuado de fármacos en animales de abasto, sin respetar las Buenas Prácticas Pecuarias (BPP), conlleva a la acumulación de residuos en los alimentos.

Los hallazgos de residuos de IVM que sobrepasan los LMR establecidos por Codex Alimentarius no son raros en la ganadería. En Brasil, Barbieri (2014) encontró que el $6.0 \%(4602 / 77056)$ y $1.4 \%(1153 / 81565)$ de hígados y músculos de bovinos, respectivamente, presentaban este tipo de residuos. De igual forma, en Colombia, Lailany et al. (2016) encontraron 2\% (1/50) y Solis et al. (2011) en México reportaron $0.4 \%$ (1/234) de hígados bovinos con residuos de IVM por encima de los LMR.
Las razones de las diferencias podrían estar asociadas al uso de IVM de larga acción, al incumplimiento del periodo de retiro y a la ausencia de normas que regulen el uso de lactonas macrocíclicas en el Perú, mientras que en otros países de Latinoamérica este grupo de fármacos forman parte del Plan Nacional para el Control de Residuos y Contaminantes en Alimentos de Origen Animal (MAPA, 2010; MinSalud, 2013; SENASICA, 2020).

La frecuencia de residuos de IVM en hígados de alpacas fue mayor a las encontradas en tejido muscular de bovinos en Nicaragua, donde el $4.4 \%$ (480/10 942) y $0.1 \%$ (39/38 143) de muestras presentaban residuos superiores al LMR establecido de $10 \mu \mathrm{g} / \mathrm{kg}$, según Pérez y Torres (2013) y Arauz (2016), 
Cuadro 1. Frecuencia de resultados positivos a residuos de ivermectina en hígado de alpaca procedentes de la provincia de Caylloma, Arequipa en $2019(n=50)$

\begin{tabular}{|c|c|c|c|}
\hline \multirow{2}{*}{ Variable } & \multirow{2}{*}{$\begin{array}{c}\text { Total de } \\
\text { muestras (n) }\end{array}$} & \multicolumn{2}{|c|}{ Muestras positivas } \\
\hline & & $\mathrm{n}$ & $\%$ \\
\hline \multicolumn{4}{|l|}{ Lugar de procedencia } \\
\hline Tocra & 18 & 2 & 11.1 \\
\hline Challhuanca & 32 & 3 & 9.4 \\
\hline \multicolumn{4}{|l|}{ Grupo etario (años) } \\
\hline$<2$ & 4 & 1 & 25.0 \\
\hline $2-3$ & 6 & 1 & 16.7 \\
\hline $3-4$ & 11 & 1 & 9.1 \\
\hline$>4$ & 29 & 2 & 6.9 \\
\hline \multicolumn{4}{|l|}{ Sexo } \\
\hline Macho & 16 & 2 & 12.5 \\
\hline Hembra & 34 & 3 & 8.8 \\
\hline \multicolumn{4}{|l|}{ Raza } \\
\hline Huacaya & 38 & 4 & 10.5 \\
\hline Suri & 12 & 1 & 8.3 \\
\hline Total & 50 & 5 & 10.0 \\
\hline
\end{tabular}

respectivamente. Se debe de tener en cuenta que Nicaragua cuenta con una normativa vigente que prohíbe el uso de IVM de larga acción en la ganadería, así como la baja afinidad del fármaco por el músculo a diferencia del hígado (Chiu et al., 1990; Mora et al., 2010).

El estudio no reportó diferencia significativa en la proporción de muestras positivas a residuos de IVM según procedencia; sin embargo, estas variaciones pueden depender de los factores culturales y de la endemicidad de las enfermedades que requieran el uso de IVM. En ese sentido, Arauz (2016) encontró una mayor frecuencia de muestras con valores elevados de residuos de IVM (35.9\%) en el departamento de Managua (Nicaragua) que en otros departamentos. Asimismo, Barbieri (2014) en Brasil encontró una mayor proporción $(11.4 \%)$ de muestras del estado de Minas Gerais con residuos de IVM que superaban los LMR que en otras partes del Brasil.

El estudio no e encontró influencia de la edad en la presentación de residuos de IVM, lo que concuerda con el trabajo de Barbieri (2014) en Brasil. Esto se explicaría por el hecho de que la infestación por ectoparásitos en alpacas se produce en todas las edades, además de que la IVM se estaría utilizando preventivamente en todo el rebaño, independientemente del estrato etario. Tampoco se encontró diferencia en la presentación de residuos de IVM por efecto del sexo y raza de las alpacas. Sin embargo, Barbieri (2014) 
encontró que los toros presentaban 8.1\% más de casos con residuos encima del LMR, lo que fue atribuido a que los toros procedían de un sistema de confinamiento, donde existe un mejor control sanitario con uso de antiparasitarios.

Los impactos negativos de la IVM han hecho que las organizaciones internacionales regulen con fundamento científico los residuos de fármacos potencialmente peligrosos (Vaca, 2003). En el caso peruano, existe la Norma Técnica Sanitaria que establece los LMR de medicamentos veterinarios en alimentos de consumo humano (NTS N. ${ }^{\circ} 120$ MINSA/DIGESA-V.01), que está basada en la normativa del Codex Alimentarius (MINSA, 2016). Según este documento, se han establecido LMR para los antibióticos, promotores de crecimiento, algunos antiparasitarios e insecticidas, pero las avermectinas no están incluidas a pesar de su uso extendido en diversas especies productivas.

En ese sentido, se hace necesario implementar un programa de vigilancia sanitaria en busca de mejorar la inocuidad de los alimentos (CAPV, 2016). Carmona y Vindas (2013) refieren que este programa debe ir junto con la capacitación de los productores y personal sanitario en temas relacionados a las Buenas Practicas Ganaderas. Muestra de su efectividad se observa en Nicaragua, donde la frecuencia de residuos por encima de LMR se redujo de $4.4 \%$ a $0.1 \%$ luego de su implementación (Pérez y Torres, 2013; Arauz, 2016).

En el presente estudio se han considerado algunas limitaciones; entre ellas, la recolección de muestras se realizó en julio y agosto, meses diferentes a los que se suele usar la IVM en forma masiva en las denominadas faenas comunales (mayo y noviembre), por lo que los resultados obtenidos podrían subestimar la prevalencia real de residuos en otros meses. Por otro lado, el kit de ELISA presenta alta reactividad cruzada con otras lactonas macrocíclicas, lo que podría haber influido en los resultados, pero que son compuestos de igual importancia en la salud pública. Los resultados deben alertar a las autoridades del Ministerio de Salud y el Servicio Nacional de Sanidad Agraria (SENASA) a fin de tomar medidas que busquen evitar futuros problemas sobre la salud pública, especialmente cuando los residuos superan en gran medida los LMR establecidos por el Codex Alimentarius. La prevención y control del problema comienza con la implementación de un sistema de vigilancia, para lo cual las avermectinas deberían ser incluidas en la Norma Sanitaria NTS N. ${ }^{\circ} 120-M I N S A /$ DIGESA-V.01, así como de manera simultánea, capacitar a los productores sobre las buenas prácticas pecuarias con fines de garantizar la inocuidad en la cadena productiva de camélidos sudamericanos en el país.

\section{Conclusiones}

- Se encontró la presencia de residuos de ivermectina (IVM) en hígado de alpacas sacrificadas en el camal del Centro Poblado de Challhuanca, Caylloma, Arequipa.

- $\quad$ El 10\% de muestras evaluadas presentaban niveles de IVM que superaban los Límites Máximos de Residuos establecidos por Codex Alimentarius.

- La procedencia, edad, raza y sexo no estuvieron asociadas a la presentación de residuos de IVM.

\section{Agradecimientos}

Los autores agradecen al Dr. Carlos Augusto San Bartolomé Gelicich (†) y al Laboratorio Multivet SRL, por la donación del kit diagnóstico para el desarrollo del presente estudio. 


\section{Literatura Citada}

1. Arauz M. 2016. Residuos de ivermectina en carne bovina en Matadero Novaterra S.A -Tipitapa enero junio 2015. Tesis de Médico Veterinario. Managua: Univ. Nacional Agraria. 43 p.

2. Barbieri C. 2014. Aspectos do controle de resíduos de avermectinas no abate de bovinos. Tese em Medicina Veterinária. Brasil: Univ. Estadual Paulista. 78 p.

3. Bioo Scientific Corporation. 2014. Avermectins ELISA Test Kit Manual 1073-01B Internet]. Available in: http:// www.biooscientific.com/MaxSignalAvermectins-ELISA-Test-Kit

4. Carmona G, Vindas $S$. 2013. Uso racional de medicamentos veterinarios en ganado bovino. Universidad Nacional de Costa Rica. 60 p. [Internet]. Disponible en: https://images.engormix.com/s articles/Carmona-Solano medicamentos.pdf

5. Casas E, Casas G, Chávez A. 2006. Evaluación de la eficacia y residualidad de una ivermectina $1.5 \%$ en vehículo de larga acción (Bovimec ${ }^{\circledR} 1.5 \%$ L.A.) en el control de la sarna en alpacas naturalmente infectadas del departamento de Cerro de Pasco. Univ. Nacional Mayor de San Marcos. [Internet]. Disponible en: http://www.agrovetmarket.com/ public/pdf/AGOSTO.07/INVESTIGACION/Bovimec\%201.5\%25\%20L.A. $\% 20$ Sarna $\% 20-$ Alpacas $\%$ 20UNMSM.pdf

6. Chiu S, Green M, Baylis F, Eline D, Rosegay A, Meriwether H, Jacob T. 1990. Absorption, tissue distribution, and excretion of tritium-labeled ivermectin in cattle, sheep, and rat. J Agr Food Chem 38: 2072-2078.

7. Codex Alimentarius. 2018. Maximum Residue Limits (MRLs) and risk management recommendations (RMRs) for residues of veterinary drugs in foods CX/MRL 2-2018. [Internet]. Disponible en: http://www.fao.org/fao-whocodexalimentarius/sh-proxy/en/
$? \operatorname{lnk}=1 \&$ url $1=$ https $\%-253 \mathrm{~A} \%$ $252 \mathrm{~F} \% 252 \mathrm{Fworkspace}$ fao.org\%$252 \mathrm{Fsites} \% 252 \mathrm{Fcodex} \% 252$ F S t and a rd s \% - 252 F C X M \% 2B2\%252FMRL2e.pdf

8. [CAPV] Comunidad Autónoma del País Vasco. 2016. Evaluación del plan de investigación de residuos en animales y sus productos en la CAPV [Internet]. Disponible en: https://www.euskadi.eus/contenidos/informacion/ cont alim seg quimica/es def/adjuntos/ Evaluacion-plan \%20-nacionalinvestigacion-residuos-CAPV-2016.pdf

9. Condemayta S, Tapia M, Apaza E. 2014. Evaluación antisárnica y antinematódica de una ivermectina de larga acción (Alpamec L.A.) en alpacas del Centro de Investigación y Producción La Raya. Puno, Perú: Univ. Nacional del Altiplano. $10 \mathrm{p}$.

10. Condori T, Ccorimanya E. 2018. Fortalecimiento de las capacidades para la producción, acopio y comercialización organizada de fibra de alpaca Challhuanca Yanque. Arequipa, Perú: Asociación Zonal de Criadores de Camélidos Andinos Challhuanca Yanque ColcaCamel. $52 \mathrm{p}$.

11. Danaher M, Howells C, Crooks H, Cerkvenik-Flajs V, O'keeffe M. 2006. Review of methodology for the determination of macrocyclic lactone residues in biological matrices. J Chromatogr A 844: 175-203. doi: 10.1016/j.jchromb.2006.07.035

12. De los Ríos E. 2010. Estado de la situación del sector textil camélidos en el Perú (Diagnóstico nacional) [Internet]. Lima: Organización de Naciones Unidas para el Desarrollo Industrial (UNIDO). 47 p.

13. [EMA] European Medicines Agency. 2014. European public MRL assessment report (EPMAR) Ivermectin (All mammalian food producing species) [Internet]. Available in: https://www.ema.europa.eu/en/documents/mrlreport/ivermectin-all-mammalian-foodproducing-species-european-publicmaximum-residue-limit-assessment_en.pdf 
14. Escribano M, San Andrés MI, de Lucas JJ, González-Canga A. 2012. Ivermectin residue depletion in food producing species and its presence in animal foodstuffs with a view to human safety. Curr Pharm Biotechnol 13: 98798. doi: 10.2174/138920112800399121

15. Fernández-Baca S. 2005. Situación actual de los camélidos sudamericanos en Perú. Proyecto de Cooperación Técnica en apoyo de la crianza y aprovechamiento de los camélidos sudamericanos en la Región Andina TCP/RLA/2914. Roma: FAO. 62 p.

16. [INEI] Instituto Nacional de Estadística e Informática. 2012. Resultados definitivos IV Censo Nacional Agropecuario. [Internet]. Disponible en https:// sinia.minam.gob.pe/documentos/resultados-definitivos-iv-censo-nacionalagropecuario-2012-0

17. Lailany D, Teresa C, Andrei S, Ordoñez D, Roa L. 2016. Estudio preliminar sobre el uso de la técnica ELISA competitiva para la detección de residuos de ivermectina en hígado de bovinos. Tesis de Médico Veterinario. Bogotá, Perú: Univ. de Ciencias Aplicadas y Ambientales. $23 \mathrm{p}$.

18. [MAPA] Ministério da Agricultura, Pecuária e Abastecimento. (29 de abril 2010). Instrução Normativa $N^{\circ} 8$. Programa de Controle de Resíduos e Contaminantes em carnes - PNCRC/2010 [internet]. [Acesso 21 abril 2020]. Brasília: MAPA. 11p.

19. [MINSA] Ministerio de Salud. 2016. Aprueban la NTS $N^{\circ} 120$-MINSA/ DIGESA-V.01 «Norma Sanitaria que establece los Límites Máximos de Residuos (LMR) de medicamentos veterinarios en alimentos de consumo humano». Diario oficial El Peruano (Lima).

20. [MinSalud] Ministerio de Salud y Protección Social. (2 de mayo 2013). Resolución $N^{\circ} 1382$. Límites máximos para residuos de medicamentos veterinarios en los alimentos de origen animal [Internet]. Disponible en: https:// www.minsalud.gov.co/sites/rid/Lists/
BibliotecaDigital/RIDE/DE/DIJ/ resolucion-1382-de-2013.pdf

21. Mora G, Liechti H, Alavarado L, Lopez L, Martinez D, Zapata M, et al. 2010. Norma técnica obligatoria nicaragüense NTON 03 087-09. Límite Máximo de Residuos de medicamentos veterinarios [Internet]. Disponible en: http://legislacion.asamblea.gob.ni/ normaweb.nsf/b92aaea 87 dac7624$06257265005 \mathrm{~d} 21 \mathrm{f} 7 / 57656 \mathrm{f} 74 \mathrm{bb} 311 \mathrm{e}-$ 360625784600565067?OpenDocument

22. Pérez F, Torres A. 2013. Residuos de ivermectina en carne bovina en industrial comercial San Martín - matadero de Nandaime septiembre 2012 - septiembre 2013. Tesis de Médico Veterinario. Managua: Univ. Nacional Agraria. 49 p.

23. Plumb C. 2010. Ivermectina. En: Manual de farmacología veterinaria. $6^{\mathrm{a}} \mathrm{ed}$. Argentina: Inter-Médica. p 622-627.

24. [SENASICA] Servicio Nacional de Sanidad, Inocuidady Calidad Agroalimentaria. 2020. Tabla de límites máximos de residuos 2020 [Internet]. Disponible en: https://www.gob.mx/cms/ uploads/attachment/file/542457/TABLA-LMR-2020.pdf

25. Shi W, He J, Jiang H, Hou X, Yang J, Shen J. 2006. Determination of multiresidue of avermectins in bovine liver by an indirect competitive ELISA. J Agric Food Chem 54: 6143-6146. doi: $10.1021 / \mathrm{jf} 060878 \mathrm{v}$

26. Solis $C$, Wilcock A, Arellano S, Morales L, Mcewen S. 2011. Prevalence of ivermectin residues in cattle slaughtered in federally inspected abattoirs in Nuevo Leon, Mexico. Food Prot Trends 31:212-215.

27. Solis $C$, Wilcock A, Arellano S, Morales L, Mcewen S. 2011. Prevalence of ivermectin residues in cattle slaughtered in federally inspected abattoirs in Nuevo León, México. Food Prot Trends 31:212-215.

28. Vaca A. 2003. Aspectos regulatorios de los medicamentos veterinarios registrados en Colombia e incluidos en el Codex Alimentarius». Tesis de Médico Veterinario. Bogotá: Univ. Nacional de Colombia. $49 \mathrm{p}$. 